Tohoku Math. J.

68 (2016), 139-159

\title{
A NOTE ON RHODES AND GOTTLIEB-RHODES GROUPS
}

\author{
Kyoung Hwan Choi, Jang Hyun Jo And Jae Min Moon
}

(Received April 25, 2014, revised October 6, 2014)

\begin{abstract}
The purpose of this paper is to give positive answers to some questions which are related to Fox, Rhodes, Gottlieb-Fox, and Gottlieb-Rhodes groups. Firstly, we show that for a compactly generated Hausdorff based $G$-space $\left(X, x_{0}, G\right)$ with free and properly discontinuous $G$-action, if $\left(X, x_{0}, G\right)$ is homotopically $n$-equivariant, then the $n$-th GottliebRhodes group $G \sigma_{n}\left(X, x_{0}, G\right)$ is isomorphic to the $n$-th Gottlieb-Fox group $G \tau_{n}\left(X / G, p\left(x_{0}\right)\right)$. Secondly, we prove that every short exact sequence of groups is $n$-Rhodes-Fox realizable for any positive integer $n$. Finally, we present some positive answers to restricted realization problems for Gottlieb-Fox groups and Gottlieb-Rhodes groups.
\end{abstract}

1. Introduction. The $n$-th Fox homotopy group or the $n$-th torus homotopy group $\tau_{n}\left(X, x_{0}\right)$ of a based space $\left(X, x_{0}\right)$ was given by Fox in 1945 to explain a geometric interpretation of the classical Whitehead product of elements of higher homotopy groups $([5,6])$. For a transformation group $(X, G)$ or a $G$-space $X$, Rhodes introduced the $n$-th Rhodes group $\sigma_{n}\left(X, x_{0}, G\right)$, where $x_{0}$ is a base point in $X$, which is an analogue of the higher homotopy groups $\pi_{n}\left(X, x_{0}\right)$. In 1983, Woo and Yoon [19] investigated Gottlieb-Rhodes groups which is a generalization of classical Gottlieb groups, and extended some results on classical Gottlieb groups to Gottlieb-Rhodes groups. Golasiński, Gonçalves, and Wong [8] in 2007 studied Gottlieb-Fox groups and Gottlieb-Rhodes groups, and presented some interesting properties of those.

The purpose of this paper is to give some positive answers to the following questions which are related to Fox groups, Rhodes groups, Gottlieb-Fox groups, and Gottlieb-Rhodes groups.

The first one is when Gottlieb-Rhodes groups of a space $X$ with free and properly discontinuous $G$-action are isomorphic to Gottlieb-Fox groups of its orbit space $X / G$. The second one is a realization problem for Rhodes groups. And the third one is about some restricted realization problems for Fox, Rhodes, Gottlieb-Fox, and Gottlieb-Rhodes groups.

In 2007, Golasiński, et al. showed in [8, Theorem 1.2] that for a finite group $G$ and a positive integer $n$, if $G$ acts freely on a based space $\left(X, x_{0}\right)$, then the Rhodes group $\sigma_{n}\left(X, x_{0}, G\right)$ of $(X, G)$ is isomorphic to the Fox group $\tau_{n}(X / G)$ of its orbit space under the isomorphism $\Phi_{n}$ induced from a regular covering projection. By the same argument as in the proof of $[8$, Theorem 1.2] we may also conclude that if an arbitrary group $G$ acts freely and properly discontinuously on a based space $\left(X, x_{0}\right)$, then for any $n \geq 1, \sigma_{n}\left(X, x_{0}, G\right)$ is isomorphic to the Fox

2010 Mathematics Subject Classification. Primary 55Q05; Secondary 55Q70.

Key words and phrases. Fox homotopy group, Gottlieb group, Gottlieb-Fox group, Gottlieb-Rhodes group, Rhodes group. 
group $\tau_{n}(X / G)$ under the isomorphism $\Phi_{n}$. Thus it is natural to ask whether $G \sigma_{n}\left(X, x_{0}, G\right)$ is isomorphic to $G \tau_{n}\left(X / G, p\left(x_{0}\right)\right)$ for all $n$ under the same isomorphism $\Phi_{n}$. But we can not expect a positive answer to this question as in Example 3.3, since $G \tau_{n}\left(X / G, p\left(x_{0}\right)\right)$ is abelian and $G \sigma_{n}\left(X, x_{0}, G\right)$ is not abelian in general. However we can show in Theorem 3.11 that for a compactly generated Hausdorff based $G$-space $\left(X, x_{0}, G\right)$ with free and properly discontinuous $G$-action, if $\left(X, x_{0}, G\right)$ is homotopically $n$-equivariant, then the $n$-th GottliebRhodes group $G \sigma_{n}\left(X, x_{0}, G\right)$ is isomorphic to the $n$-th Gottlieb-Fox group $G \tau_{n}\left(X / G, p\left(x_{0}\right)\right)$. As a byproduct, we can conclude that for a compactly generated Hausdorff based $G$-space $\left(X, x_{0}, G\right)$ with free and properly discontinuous $G$-action, if $\left(X, x_{0}, G\right)$ is homotopically $n$ equivariant, then $G \sigma_{n}\left(X, x_{0}, G\right)$ is abelian.

In order to give an affirmative answer to the second question, we apply the result of Lokutsievskii [13]. Recall that if a group $G$ acts freely and properly discontinuously on a topological space $X$, then $p: X \rightarrow X / G$ is a regular covering projection and there is a group extension $1 \rightarrow \pi_{1}(X) \rightarrow \pi_{1}(X / G) \rightarrow G \rightarrow 1$. It is natural to ask whether the converse of this holds, i.e., given any group extension $1 \rightarrow N \rightarrow \Gamma \rightarrow G \rightarrow 1$, there exits a topological space $X$ on which $G$ acts freely and proper discontinuously such that the extension $1 \rightarrow N \rightarrow$ $\Gamma \rightarrow G \rightarrow 1$ is isomorphic to $1 \rightarrow \pi_{1}(X) \rightarrow \pi_{1}(X / G) \rightarrow G \rightarrow 1$. The positive answer to this question seems to be well-known or a folklore. But we can not find any reference except Lokutsievskii's paper. In [13], Lokutsievskii presented these in very details. In fact, he showed that given an extension of groups $1 \rightarrow N \rightarrow \Gamma \rightarrow G \rightarrow 1$ with finite $G$, there is a $K(N, 1)$ complex $X$ with a free $G$-action such that $1 \rightarrow \pi_{1}(X) \rightarrow \pi_{1}(X / G) \rightarrow G$ is isomorphic to the given extension. Moreover, he proved that there is a one to one correspondence between the set of all free actions up to homotopy conjugation of $G$ on $K(N, 1)$-complexes and the set of all classes of isomorphic extensions of $N$ by $G$. Without any difficulty we have the same result of his for the case of arbitrary group extensions. Using this result, we show in Theorem 4.8 that every short exact sequence of groups is $n$-Rhodes-Fox realizable for any positive integer $n$, more precisely, for any extension of groups $1 \rightarrow N \rightarrow \Gamma \rightarrow G \rightarrow 1$ and any positive integer $n$, there is a $K(N, 1)$-complex $X$ with free $G$-action such that for any positive integer $n$, the exact sequence of groups $1 \rightarrow \tau_{n}(X) \rightarrow \sigma_{n}(X, G) \rightarrow G \rightarrow 1$ is isomorphic to $1 \rightarrow N \rightarrow \Gamma \rightarrow G \rightarrow 1$.

For the third question, recall the following question posed by Oprea and Strom [15]: for a finitely generated abelian group $\Gamma$ and a positive integer $n$, is there a finite $C W$-complex or a compact manifold $X$ with $G_{n}(X) \cong \Gamma$ ? They proved that for any finitely generated abelian group $\Gamma$, there exists a compact manifold $X$ with $G_{1}(X) \cong \Gamma$. For $n>1$, they proved that for a finite abelian group $\Gamma$, there is an $N$ so that for each $n \geq N$ there is a compact manifold $X$ with $\Gamma$ isomorphic to a subgroup of $G_{2 n}(X)$. Moreover they showed using rational homotopy theory that for any even number $n$, any torsion-free abelian group cannot be realized as $G_{n}(X)$ for a finite complex $X$.

It can be seen in Lemma 4.14 that if $\Gamma$ is an arbitrary abelian group and $n$ is any positive integer, then $\tau_{n}(K(\Gamma, n))=G \tau_{n}(K(\Gamma, n)) \cong \Gamma$. Thus if there is no restrictions on spaces, then the third question about Fox-groups and Gottlieb-Fox groups is trivial like the ordinary 
Gottlieb groups ([15]). In Theorems 4.15 and 4.16, we prove that for a finitely generated abelian group $\Gamma$ and any positive integer $n$, there exists a compact manifold $X$ which is a Gottlieb-Fox space such that $\tau_{n}(X)=G \tau_{n}(X) \cong \Gamma$. Also we show in Theorem 4.18 that if $\Gamma$ is a finitely generated abelian group, $N$ is a free abelian subgroup of $\Gamma$, and $G$ is a torsion subgroup of $\Gamma$, then for any positive integer $n$, there exists a compact $G$-manifold $X$ such that the group extension $1 \rightarrow G \tau_{n}(X) \rightarrow G \sigma_{n}(X, G) \rightarrow G \rightarrow 1$ is isomorphic to $1 \rightarrow N \rightarrow \Gamma \rightarrow G \rightarrow 1$.

2. Preliminaries. Through out this paper, every group is a discrete group and all spaces considered are supposed to be topological spaces.

In this section, we collect some basic properties and definitions of Fox, Rhodes, Gottlieb, Gottlieb-Fox, and Gottlieb-Rhodes groups. We also briefly recall some miscellaneous facts which we will used in the main results. For more details, we recommend corresponding references.

1. ([6, 7]) The $n$-th Fox homotopy group or the $n$-th torus homotopy group of a based space $\left(X, x_{0}\right)$ is defined to be $\tau_{n}\left(X, x_{0}\right):=\pi_{1}\left(X^{T^{n-1}}, c_{x_{0}}\right)$, where $X^{T^{n-1}}$ is the space of continuous maps from the $(n-1)$-dimensional torus $T^{n-1}$ to $X$ with the compact open topology and $c_{x_{0}}$ is the constant map based at $x_{0}$. It is clear that $\tau_{1}\left(X, x_{0}\right)=\pi_{1}\left(X, x_{0}\right)$. It can be seen that for a path connected space $X, \tau_{n}\left(X, x_{0}\right)$ is independent of the base point $x_{0}$ up to isomorphism groups. Thus $\tau_{n}\left(X, x_{0}\right)$ is usually simply denoted by $\tau_{n}(X)$ for a path connected space $X$. The following are well-known facts concerning Fox homotopy groups:

(a) For any positive integer $n \geq 2$, the sequence

$$
1 \rightarrow \prod_{i=2}^{n} \pi_{i}\left(X, x_{0}\right)^{\alpha_{i}} \rightarrow \tau_{n}\left(X, x_{0}\right) \rightarrow \tau_{n-1}\left(X, x_{0}\right) \rightarrow 1
$$

is split exact, where $\alpha_{i}$ is the binomial coefficient $\left(\begin{array}{c}n-2 \\ i-2\end{array}\right)$.

Moreover, $\prod_{i=2}^{n} \pi_{i}\left(X, x_{0}\right)^{\alpha_{i}} \cong \tau_{n-1}\left(\Omega X, c_{x_{0}}\right)$, where $\Omega X$ is the loop space of $X$ and $c_{x_{0}}$ is the constant map based at $x_{0}$.

(b) The Whitehead product of two elements $\alpha \in \pi_{m}\left(X, x_{0}\right), \beta \in \pi_{n}\left(X, x_{0}\right)$ in $\pi_{m+n-1}(X$, $\left.x_{0}\right)$ is a commutator when $\pi_{m+n-1}\left(X, x_{0}\right)$ is embedded in $\tau_{k}\left(X, x_{0}\right)$ for $k \geq m+n+1$.

In [7] Golasiński et al. reformulate the definition of Fox homotopy groups and investigate generalized Fox homotopy groups.

2. For a transformation group $(X, G)$ or a $G$-space $X$, the $n$-th Rhodes group $\sigma_{n}\left(X, x_{0}\right.$, $G)$, where $x_{0}$ is a base point in $X$, is defined to be

$$
\left.\bigcup_{g \in G}\left\{[f ; g] \mid f: I \times T^{n-1} \rightarrow X, f\left(0, t_{1}, \ldots, t_{n-1}\right)=x_{0}, f\left(1, t_{1}, \ldots, t_{n-1}\right)=g \cdot x_{0}\right)\right\}
$$

with the operation $\left[f_{1} ; g_{1}\right] *\left[f_{2} ; g_{2}\right]:=\left[f_{1}+g_{1} \cdot f_{2} ; g_{1} g_{2}\right]$. It is clear that $\tau_{n}\left(X, x_{0}\right) \cong$ $\sigma_{n}\left(X, x_{0},\{e\}\right)$. It can be seen that for a path connected $G$-space $X, \tau_{n}\left(X, x_{0}, G\right)$ is independent of the base point $x_{0}$ up to isomorphism groups. Thus $\sigma_{n}\left(X, x_{0}, G\right)$ is usually simply denoted by $\sigma_{n}(X, G)$ for a path connected $G$-space $X$. It is well-known that the sequence $1 \rightarrow \tau_{n}\left(X, x_{0}\right) \rightarrow \sigma_{n}\left(X, x_{0}, G\right) \rightarrow G \rightarrow 1$ is exact. 
3. ([10,11]) Let $\left(X, x_{0}\right)$ be a based space. Consider the class of continuous maps $F$ : $X \times S^{n} \rightarrow X$ such that $F\left(x, s_{0}\right)=x$, where $x \in X$ and $s_{0}$ is a base point of $S^{n}$. Then the map $f: S^{n} \rightarrow X$ defined by $f(s):=F\left(x_{0}, s\right)$, where $x_{0}$ is a base point of $X$, represents an element $[f] \in \pi_{n}\left(X, x_{0}\right)$. The set of all elements $[f] \in \pi_{n}\left(X, x_{0}\right)$ obtained by the above manner from some $F$ forms a subgroup of $\pi_{n}\left(X, x_{0}\right)$, which is called the $n$-th Gottlieb group $G_{n}\left(X, x_{0}\right)$, and the map $F$ is said to be an associated or affiliated map to $f$. For a compactly generated Hausdorff space $X$, it can be seen that $G_{n}\left(X, x_{0}\right)$ is the same as the evaluation subgroup of $\pi_{n}\left(X, x_{0}\right)$, i.e., $G_{n}\left(X, x_{0}\right)=\operatorname{Im}\left(e v_{*}: \pi_{n}\left(X^{X}, 1_{X}\right) \rightarrow \pi_{n}\left(X, x_{0}\right)\right)$. By definition, a path connected space $X$ is $n$-Gottlieb if $G_{n}(X)=\pi_{n}(X)$. If $X$ is $n$-Gottlieb for all $n \geq 1$, then it is said to be Gottlieb. It is well-known that any $H$-space is a Gottlieb space.

4. ([7, 8, 9, 19]) Let $X$ be a $G$-space. For any $[f ; g] \in \sigma_{n}\left(X, x_{0}, G\right)$, a continuous map $H: X \times I \times T^{n-1} \rightarrow X$ is said to be an associated or affiliated map to $f$ if $H\left(x, 0, t_{1}, \ldots, t_{n-1}\right)=x, H\left(x, 1, t_{1}, \ldots, t_{n-1}\right)=g \cdot x, H\left(x_{0}, s, t_{1}, \ldots, t_{n-1}\right)=f\left(s, t_{1}, \ldots\right.$, $\left.t_{n-1}\right)$. The $n$-th Gottlieb-Rhodes group $G \sigma_{n}\left(X, x_{0}, G\right)$ is defined to be the subgroup of $\sigma_{n}(X$, $\left.x_{0}, G\right)$ which consists of elements $[f ; g]$ such that $f$ has an associated map. For a compactly generated Hausdorff space $X$ with $G$-action, it can be seen that $G \sigma_{n}\left(X, x_{0}, G\right)$ is the same as the evaluation subgroup of $\sigma_{n}\left(X, x_{0}, G\right)$, i.e., $G \sigma_{n}\left(X, x_{0}, G\right)=\operatorname{Im}\left(e v_{*}: \sigma_{n}\left(X^{X}, 1_{X}, G\right) \rightarrow\right.$ $\left.\sigma_{n}\left(X, x_{0}, G\right)\right)$. For a based space $\left(X, x_{0}\right)$, if we consider $X$ as $X$ with trivial $G$-action, then the $n$-th Gottlieb-Fox group $G \tau_{n}\left(X, x_{0}\right)$ is defined to be $G \sigma_{n}\left(X, x_{0},\{e\}\right)$. A path connected space $X$ is said to be $n$-Gottlieb-Fox if $G \tau_{n}(X)=\tau_{n}(X)$, and a path connected $G$-space $X$ is said to be $n$-Gottlieb-Rhodes if $G \sigma_{n}(X, G)=\sigma_{n}(X, G)$. By definition, a path connected space $X$ is Gottlieb-Fox if it is $n$-Gottlieb-Fox for all $n \geq 1$ and a path connected $G$-space $X$ is GottliebRhodes if it is $n$-Gottlieb-Rhodes or all $n \geq 1$. The following facts about Gottlieb-Fox and Gottlieb-Rhodes groups have been shown in [7, 8, 9]:

(a) For a compactly generated Hausdorff space $X$ with $G$-action, the sequence $1 \rightarrow$ $G \tau_{n}\left(X, x_{0}\right) \rightarrow G \sigma_{n}\left(X, x_{0}, G\right) \rightarrow G_{0} \rightarrow 1$ is exact, where $G_{0}$ is the subgroup of $G$ consisting of elements $g$ considered as homeomorphisms of $X$ which are freely homotopic to $1_{X}$.

(b) For a compactly generated Hausdorff space $X$ and an integer $n \geq 2$, there is a split exact sequence of groups:

$$
1 \rightarrow G \tau_{n-1}\left(\Omega X, c_{x_{0}}\right) \rightarrow G \tau_{n}\left(X, x_{0}\right) \rightarrow G \tau_{n-1}\left(X, x_{0}\right) \rightarrow 1,
$$

where $\Omega X$ is the loop space of $X$ and $c_{x_{0}}$ is the constant map based at $x_{0}$. Moreover $G \tau_{n-1}$ $\left(\Omega X, c_{x_{0}}\right) \cong \prod_{i=2}^{n} G_{i}\left(X, x_{0}\right)^{\alpha_{i}}$, where $\alpha_{i}:=\left(\begin{array}{c}n-2 \\ i-2\end{array}\right)$, and $G \tau_{n}\left(X, x_{0}\right) \cong \prod_{i=2}^{n} G_{i}\left(X, x_{0}\right)^{\gamma_{i}}$, where $\gamma_{i}:=\left(\begin{array}{c}n-1 \\ i-1\end{array}\right)$.

(c) For a compactly generated Hausdorff space $X$ and any positive integer $n, G \tau_{n}\left(X, x_{0}\right)$ is central in $\tau_{n}\left(X, x_{0}\right)$ so that $G \tau_{n}\left(X, x_{0}\right)$ is abelian. But for a compactly generated Hausdorff space $X$ with $G$-action, $G \sigma_{n}\left(X, x_{0}, G\right)$ is not necessarily abelian in general.

(d) For all $k \leq n, \tau_{k}\left(S^{n}\right) \cong \pi_{k}\left(S^{n}\right)$.

(e) Let $G$ be any abelian group and $n \geq 2$. For any $K(G, n)$-complex $X$, if $k<n$ then $\tau_{k}(X)=1$, and if $k \geq n$ then $\tau_{k}(X) \cong G^{(k-1)}$. In particular, $\tau_{k}(K(G, 1)) \cong G$ for all $k \geq 1$. 
(f) A compactly generated Hausdorff path connected space $X$ is $n$-Gottlieb if and only if it is $n$-Gottlieb-Fox.

5. ([16, 17, 20]) By definition, a transformation group $(X, G)$ admits a family of preferred paths at $x_{0}$ if it is possible to associate with every element $g_{1}$ of $G$ a path $k_{1}$ from $g_{1} \cdot x_{0}$ to $x_{0}$ such that the path $k_{0}$ associated with the identity element $e$ is $c_{x_{0}}$ and for every pair of elements $g_{1}, g_{2}$ the path $k_{1,2}$ from $g_{1} g_{2} \cdot x_{0}$ to $x_{0}$ is homotopic to $g_{1} \cdot k_{2}+k_{1}$. For a transformation group $(X, G)$ and $g \in G$, a continuous map $H: X \times I \rightarrow X$ is said to be a homotopy of order $g$ if $H(x, 0)=x, H(x, 1)=g \cdot x$. A family $K$ of preferred paths at $x_{0}$ is called a family of preferred traces at $x_{0}$ if for every preferred path $k_{g}$ in $K, \overline{k_{g}}$ is the trace of a homotopy of order $g$, where $\overline{k_{g}}$ is the inverse path of $k_{g}$. The following shows that why the notions of preferred paths or preferred traces are important.

(a) The transformation group $(X, G)$ admits a family of preferred paths at $x_{0}$ if and only if $\sigma_{n}\left(X, x_{0}, G\right)$ is a split extension of $\tau_{n}\left(X, x_{0}\right)$ by $G$.

(b) If the transformation group $(G, G)$ admits a family of preferred paths at $e$, then $\sigma_{n}\left(X, x_{0}, G\right) \cong \tau_{n}\left(X, x_{0}\right) \times G$.

(c) $(X, G)$ admits a family of preferred traces at $x_{0}$

if and only if $G \sigma_{1}\left(X, x_{0}, G\right) \cong G \tau_{1}\left(X, x_{0}\right) \rtimes G_{0}$

if and only if $G \sigma_{1}\left(X, x_{0}, G\right) \cong G \tau_{1}\left(X, x_{0}\right) \times G_{0}$.

6. ([4, 18]) By definition, a group $G$ is said to act properly discontinuously on a space $X$ if every point $x \in X$ has a neighborhood $U$ such that $\{g \in G \mid g \cdot U \cap U \neq \emptyset\}$ is finite. It is well-known that $(a)$ if $G$ acts freely and properly discontinuously on a path connected Hausdorff space $X$, then $p: X \rightarrow X / G$ is a regular covering projection (b) if $G$ acts freely on a connected $C W$-complex $X$, then $G$ acts also properly discontinuously on $X$.

7. (cf. [4]) By definition, an extension of a group $N$ by a group $G$ is a short exact sequence of groups $1 \rightarrow N \rightarrow \Gamma \rightarrow G \rightarrow 1$. Two group extensions $1 \rightarrow N_{1} \rightarrow \Gamma_{1} \rightarrow$ $G_{1} \rightarrow 1$ and $1 \rightarrow N_{2} \rightarrow \Gamma_{2} \rightarrow G_{2} \rightarrow 1$ are said to be isomorphic if there are isomorphisms $N_{1} \rightarrow N_{2}, G_{1} \rightarrow G_{2}$ and $\Gamma_{1} \rightarrow \Gamma_{2}$ such that the following diagram commutes:

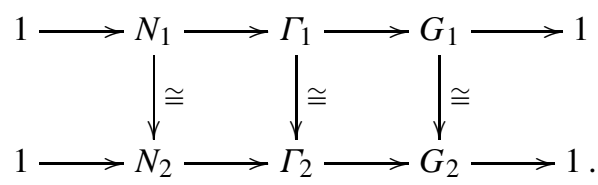

3. When are Gottlieb-Rhodes groups of a compactly generated Hausdorff space $X$ with free and properly discontinuous $G$-action isomorphic to Gottlieb-Fox groups of its orbit space $X / G$ ? In this section we investigate the question stated in the title above. Let $G$ be a group which acts freely and properly discontinuously on a space $X$ with a base point $x_{0}$. Then $p: X \rightarrow X / G$ be a regular covering projection. In 2007, Golasiński, et al. [8] showed that for a finite group $H$ and any positive integer $n$, if $H$ acts freely on a space $Y$, then the Rhodes group $\sigma_{n}\left(Y, y_{0}, H\right)$ of $(Y, H)$ is isomorphic to the Fox group $\tau_{n}(Y / H)$ of its orbit space under the map $\Phi_{n}$ below which is induced from the regular covering projection $p$. 
They used the result of Armstrong [3] and the mathematical induction. Since the proof given in [8] for the case of finite groups works in general, we have the following theorem.

THEOREM 3.1 (cf. [8, Theorem 1.2]). Let $G$ be a group which acts freely and properly discontinuously on a based space $\left(X, x_{0}\right)$. Then

$$
\Phi_{n}: \sigma_{n}\left(X, x_{0}, G\right) \rightarrow \tau_{n}\left(X / G, p\left(x_{0}\right)\right)
$$

defined by $\Phi_{n}([f ; g])=[p \circ f]$ is an isomorphism for all $n \geq 1$, where $p: X \rightarrow X / G$ is a regular covering projection.

Due to Theorem 3.1 it can be naturally asked whether $G \sigma_{n}\left(X, x_{0}, G\right)$ is isomorphic to $G \tau_{n}\left(X / G, p\left(x_{0}\right)\right)$ for all $n$ under the same isomorphism $\Phi_{n}$. But we can not expect a positive answer to this question, since $G \tau_{n}\left(X / G, p\left(x_{0}\right)\right)$ is abelian and $G \sigma_{n}\left(X, x_{0}, G\right)$ is not necessarily abelian in general. We will give an example which shows that $G \sigma_{n}\left(X, x_{0}, G\right)$ is not isomorphic to $G \tau_{n}\left(X / G, p\left(x_{0}\right)\right)$. Other examples can be founded in [8, Example 2.3].

LEMMA 3.2 ([8, Example 2.6]). Let a finite group $G$ act freely on an odd dimensional sphere $X=S^{2 n+1}$. Then $G=G_{0}$, where $G_{0}$ is the subgroup of $G$ consisting elements $g$ considered as homeomorphisms of $X$ which are freely homotopic to $1_{X}$.

PROOF. It follows from the Lefschetz fixed point theorem (cf. [12]) and the Hopf classification theorem (cf. [12]) as explained in [8, Example 2.6].

EXAMPLE 3.3. It is well-known that a finite group $G$ acts freely on some sphere if and only if $G$ satisfies both the $p^{2}$ and the $2 p$ condition for all primes $p$, i.e., every subgroup of order $p^{2}$ or $2 p$ is cyclic (cf. [1]). Let $X$ be an odd dimensional sphere $S^{3}$ or $S^{7}$, and let $G$ be a nonabelian group which acts freely on $X$. For instance, if $G$ is the generalized quaternion group, then $G$ acts freely on $S^{3}$. Note that $G=G_{0}$ by Lemma 3.2. Since $S^{3}$ and $S^{7}$ are $H$-spaces, they are Gottlieb-Fox and so $G \tau_{1}(X)=\tau_{1}(X)=\{1\}$. Since $1 \rightarrow G \tau_{1}(X) \rightarrow$ $G \sigma_{1}(X, G) \rightarrow G \rightarrow 1$ is exact, it follows that $G \sigma_{1}(X, G) \cong G$. Since $G \tau_{1}(X / G)$ is abelian, $G \sigma_{1}(X, G)$ can not be isomorphic to $G \tau_{1}(X / G)$.

The $n=1$ case of the following proposition was proved in [19, Theorem 1.2]. To help to understand Definition 3.5 and Remark 3.6 we give a proof of the following proposition which is along the lines of the proof of [19, Theorem 1.2].

Proposition 3.4. Let $\left(X, x_{0}, G\right)$ be a based $G-C W$-complex. Suppose that there exists an associated map $H: X \times I \times T^{n-1} \rightarrow X$ such that

$$
\begin{aligned}
H\left(x, 0, t_{1}, \ldots, t_{n-1}\right) & =x \\
H\left(x, 1, t_{1}, \ldots, t_{n-1}\right) & =g \cdot x \\
H\left(x_{0}, s, t_{1}, \ldots, t_{n-1}\right) & =f\left(s, t_{1}, \ldots, t_{n-1}\right) .
\end{aligned}
$$

Then for any $\left[f^{\prime} ; g\right] \in G \sigma_{n}\left(X, x_{0}, G\right)$ with $[f ; g]=\left[f^{\prime} ; g\right]$, there exists also an associated map $H^{\prime}: X \times I \times T^{n-1} \rightarrow X$ such that $H^{\prime}\left(x, 0, t_{1}, \ldots, t_{n-1}\right)=x, H^{\prime}\left(x, 1, t_{1}, \ldots, t_{n-1}\right)=$ $g \cdot x$, and $H^{\prime}\left(x_{0}, s, t_{1}, \ldots, t_{n-1}\right)=f^{\prime}\left(s, t_{1}, \ldots, t_{n-1}\right)$. 
Proof. Let $F: I \times T^{n-1} \times I \rightarrow X$ be a homotopy between $f$ and $f^{\prime}$ such that

$$
\begin{aligned}
& F\left(s, t_{1}, \ldots, t_{n-1}, 0\right)=f\left(s, t_{1}, \ldots, t_{n-1}\right), \\
& F\left(s, t_{1}, \ldots, t_{n-1}, 1\right)=f^{\prime}\left(s, t_{1}, \ldots, t_{n-1}\right), \\
& F\left(0, t_{1}, \ldots, t_{n-1}, t\right)=x_{0}, \\
& F\left(1, t_{1}, \ldots, t_{n-1}, t\right)=g \cdot x_{0} .
\end{aligned}
$$

Let $Z$ be the subcomplex of $X \times I \times T^{n-1}$ given by $\left(X \times 0 \times T^{n-1}\right) \cup\left(X \times 1 \times T^{n-1}\right) \cup$ $\left(x_{0} \times I \times T^{n-1}\right)$. Define a homotopy $K: Z \times I \rightarrow X$ on $Z$ as follows:

$$
\begin{aligned}
K\left(x, 0, t_{1}, \ldots, t_{n-1}, t\right) & :=x \\
K\left(x, 1, t_{1}, \ldots, t_{n-1}, t\right) & :=g \cdot x \\
K\left(x_{0}, s, t_{1}, \ldots, t_{n-1}, t\right) & :=F\left(s, t_{1}, \ldots, t_{n-1}, t\right) .
\end{aligned}
$$

Note that $\left.K\right|_{Z \times 0}=\left.H\right|_{Z}$. By the homotopy extension property, there exists a homotopy $L: X \times I \times T^{n-1} \times I \rightarrow X$ on $X$ such that $L(-,-,-, 0)=H$ and $\left.L(-,-,-, t)\right|_{Z \times I}=K$. Then it can be easily seen that $H^{\prime}:=L(-,-,-, 1): X \times I \times T^{n-1} \rightarrow X$ is an associated map to $f^{\prime}$.

Definition 3.5. A based $G$-space $\left(X, x_{0}, G\right)$ with free and properly discontinuous $G$-action is said to be homotopically n-equivariant if it satisfies the following: for any homotopy class $[f ; g] \in \sigma_{n}\left(X, x_{0}, G\right),[f ; g] \in G \sigma_{n}\left(X, x_{0}, G\right)$ if and only if there exist $\left[f^{\prime} ; g\right] \in G \sigma_{n}\left(X, x_{0}, G\right)$ with $[f ; g]=\left[f^{\prime} ; g\right]$ and an associated $G$-map $H: X \times I \times$ $T^{n-1} \rightarrow X$ to $f^{\prime}$ such that $H\left(x, 0, t_{1}, \ldots, t_{n-1}\right)=x, H\left(x, 1, t_{1}, \ldots, t_{n-1}\right)=g \cdot x$, and $H\left(x_{0}, s, t_{1}, \ldots, t_{n-1}\right)=f^{\prime}\left(s, t_{1}, \ldots, t_{n-1}\right)$. Here $G$ acts trivially on $I \times T^{n-1}$. If $\left(X, x_{0}, G\right)$ is homotopically $n$-equivariant for any $n \geq 1$, then we say that it is homotopically equivariant.

REMARK 3.6. Let $\left(X, x_{0}, G\right)$ be a based $G$-space with free and properly discontinuous $G$-action and $[f ; g] \in G \sigma_{n}\left(X, x_{0}, G\right)$. Then there exists an associated map $H: X \times$ $I \times T^{n-1} \rightarrow X$ to $f$ such that $H\left(x, 0, t_{1}, \ldots, t_{n-1}\right)=x, H\left(x, 1, t_{1}, \ldots, t_{n-1}\right)=g \cdot x$, and $H\left(x_{0}, s, t_{1}, \ldots, t_{n-1}\right)=f\left(s, t_{1}, \ldots, t_{n-1}\right)$. Suppose that the map $H$ is a $G$-map. Let $\left[f^{\prime} ; g\right] \in G \sigma_{n}\left(X, x_{0}, G\right)$ with $[f ; g]=\left[f^{\prime} ; g\right]$. Suppose that $f$ and $f^{\prime}$ are $G$-homotopy equivalent. Let $\mathcal{Z}$ be the subcomplex of $X \times I \times T^{n-1}$ given by $\left(X \times 0 \times T^{n-1}\right) \cup(X \times 1 \times$ $\left.T^{n-1}\right) \cup\left(x_{0} \times I \times T^{n-1}\right) \cup\left(\bigcup_{g \in G} g \cdot x_{0} \times I \times T^{n-1}\right)$. Define a homotopy $\mathcal{K}: \mathcal{Z} \times I \rightarrow X$ on $Z$ as follows:

$$
\begin{aligned}
\mathcal{K}\left(x, 0, t_{1}, \ldots, t_{n-1}, t\right) & :=x, \\
\mathcal{K}\left(x, 1, t_{1}, \ldots, t_{n-1}, t\right) & :=g \cdot x, \\
\mathcal{K}\left(x_{0}, s, t_{1}, \ldots, t_{n-1}, t\right) & :=F\left(s, t_{1}, \ldots, t_{n-1}, t\right), \\
\mathcal{K}\left(g \cdot x_{0}, s, t_{1}, \ldots, t_{n-1}, t\right) & :=g \cdot F\left(s, t_{1}, \ldots, t_{n-1}, t\right) \text { for any } g \in G .
\end{aligned}
$$

If we apply $\mathcal{Z}$ and $\mathcal{K}$ to $Z$ and $K$, respectively, in the proof of Proposition 3.4, then we may see from the $G$-homotopy extension property (cf. [2]) that $\left[f^{\prime} ; g\right]$ has also an associated $G$-map $H^{\prime}$. But it is not necessarily that $f$ and $f^{\prime}$ are $G$-homotopy equivalent. Thus in Definition 3.5 
we require that for any $[f ; g] \in G \sigma_{n}\left(X, x_{0}, G\right)$, there exists an associated $G$-map $H$ to $f^{\prime}$, where $[f ; g]=\left[f^{\prime} ; g\right]$. When we consider the notion of homotopically $n$-equivariantness, we may assume that representations of homotopy classes $[f ; g] \in G \sigma_{n}\left(X, x_{0}, G\right)$ have an associated $G$-map.

It can be seen from the argument of the proof of [10, Theorem I.3] or [19, Theorem 1.3] that for any $G-C W$-complex $X$, if $\lambda: I \rightarrow X$ is a path from $x_{0} \in X$ to $x_{1} \in X$, then $\lambda$ induces an isomorphism

$$
\lambda_{*}: G \sigma_{n}\left(X, x_{0}, G\right) \stackrel{\cong}{\rightarrow} G \sigma_{n}\left(X, x_{1}, G\right)
$$

such that $\lambda_{*}([f ; g])=\left[\lambda^{n} \rho^{n}+f+g \lambda^{n} ; g\right]$, where $\rho^{n}: I \times T^{n-1} \rightarrow I \times T^{n-1}$ is a map defined by $\rho^{n}\left(s, t_{1}, \ldots, t_{n-1}\right):=\left(1-s, t_{1}, \ldots, t_{n-1}\right)$ and $\lambda^{n}: I \times T^{n-1} \rightarrow X$ is a map defined by $\lambda^{n}\left(s, t_{1}, t_{2}, \ldots, t_{n-1}\right):=\lambda(s)$. By the similar argument, we can show the following proposition which says that the notion of homotopically $n$-equivariant is independent of the base point for a path connected free $G-C W$-complex.

Proposition 3.7. Let $X$ be a path connected $C W$-complex with free $G$-action and $n$ a positive integer. If $\left(X, x_{0}, G\right)$ is homotopically $n$-equivariant for some $x_{0} \in X$, then $\left(X, x_{1}, G\right)$ is homotopically n-equivariant for any $x_{1} \in X$.

Proof. For any point $x_{1} \in X$, let $\lambda$ be a path from $x_{0}$ to $x_{1}$. Let $\left[f^{\prime} ; g\right] \in G \sigma_{n}\left(X, x_{1}, G\right)$. Since $\lambda_{*}: G \sigma_{n}\left(X, x_{0}, G\right) \rightarrow G \sigma_{n}\left(X, x_{1}, G\right)$ is an isomorphism, there exists $[f ; g] \in$ $G \sigma_{n}\left(X, x_{0}, G\right)$ such that $\left[f^{\prime} ; g\right]=\left[\lambda^{n} \rho^{n}+f+g \lambda^{n} ; g\right]$. Since $\left(X, x_{0}, G\right)$ is homotopically $n$-equivariant, it follows from Remark 3.6 that there exist an associated $G$-map $H$ : $X \times I \times T^{n-1} \rightarrow X$ to $f$ such that $H\left(x, 0, t_{1}, \ldots, t_{n-1}\right)=x, H\left(x, 1, t_{1}, \ldots, t_{n-1}\right)=g \cdot x$, and $H\left(x_{0}, s, t_{1}, \ldots, t_{n-1}\right)=f\left(s, t_{1}, \ldots, t_{n-1}\right)$. Consider a map $p: X \times T^{n-1} \rightarrow X$ defined by $p\left(x, t_{1}, \ldots, t_{n-1}\right):=x$. It is clear that $p$ is a $G$-map. Define $A:=\bigcup_{g \in G}\left\{g \cdot x_{1}\right\}$. It is clear that $A \times I \times T^{n-1}$ is a $G-C W$-subcomplex of $X \times I \times T^{n-1}$. Define $F: A \times I \times T^{n-1} \rightarrow X$ by $F\left(g \cdot x_{1}, s, t_{1}, \ldots, t_{n-1}\right):=g \cdot \lambda^{n} \rho^{n}\left(s, t_{1}, \ldots, t_{n-1}\right)$ for any $g \in G$. It is clear that for any $\left(g \cdot x_{1}, t_{1}, \ldots, t_{n-1}\right) \in A \times T^{n-1}, F\left(g \cdot x_{1}, s, t_{1}, \ldots, t_{n-1}\right)=g \cdot x_{1}$ so that $\left.F\right|_{A \times\{0\} \times T^{n-1}}=$ $\left.p\right|_{A \times\{0\} \times T^{n-1}}$, and $F$ is a $G$-map. By the $G$-homotopy extension property, there is a $G$ homotopy $K: X \times I \times T^{n-1} \rightarrow X$ such that $\left.K\right|_{X \times\{0\} \times T^{n-1}}=p$ and $\left.K\right|_{A \times I \times T^{n-1}}=F$. Thus for any $x \in X, K\left(x, s, t_{1}, \ldots, t_{n-1}\right)=x$ and $K\left(x_{1}, s, t_{1}, \ldots, t_{n-1}\right)=\lambda^{n} \rho^{n}\left(s, t_{1}, \ldots, t_{n-1}\right)$. Define $L: X \times I \times T^{n-1} \rightarrow X$ by $L\left(x, s, t_{1}, \ldots, t_{n-1}\right):=$

$$
\begin{cases}K\left(x, 3 s, t_{1}, \ldots, t_{n-1}\right) & \text { if } 0 \leq s \leq \frac{1}{3} \\ H\left(K\left(x, 1, t_{1}, \ldots, t_{n-1}\right), 3 s-1, t_{1}, \ldots, t_{n-1}\right) & \text { if } \frac{1}{3} \leq s \leq \frac{2}{3} \\ g \cdot K\left(x, 3(1-s), t_{1}, \ldots, t_{n-1}\right) & \text { if } \frac{2}{3} \leq s \leq 1 .\end{cases}
$$

It is clear that $L$ is a $G$-map such that

$$
L\left(x_{1}, s, t_{1}, \ldots, t_{n}\right)=\left(\lambda^{n} \rho^{n}+f+g \lambda^{n}\right)\left(s, t_{1}, \ldots, t_{n}\right) \in G \sigma_{n}\left(X, x_{1}, G\right) .
$$

Hence $L$ is an associated $G$-map to $f^{\prime}$ so that $\left(X, x_{1}, G\right)$ is homotopically $n$-equivariant. 
PROPOSITION 3.8. Let $G$ be a group which acts freely and properly discontinuously on a space $X$ with a base point $x_{0}$. If $\left(X, x_{0}, G\right)$ is homotopically $n$-equivariant, then it is homotopically $k$-equivariant for every $1 \leq k \leq n$.

Proof. Let $k$ be an integer with $1 \leq k \leq n$ and $[f ; g] \in G \sigma_{k}\left(X, x_{0}, G\right)$. Then there exists an associated map $H: X \times I \times T^{k-1} \rightarrow X$ to $f$ such that

$$
\begin{aligned}
H\left(x, 0, t_{1}, \ldots, t_{k-1}\right) & =x \\
H\left(x, 1, t_{1}, \ldots, t_{k-1}\right) & =g \cdot x \\
H\left(x_{0}, s, t_{1}, \ldots, t_{k-1}\right) & =f\left(s, t_{1}, \ldots, t_{k-1}\right) .
\end{aligned}
$$

Define a map $\bar{f}: I \times T^{n-1} \longrightarrow X$ by $\bar{f}\left(s, t_{1}, \ldots, t_{n-1}\right):=f\left(s, t_{1}, \ldots, t_{k-1}\right)$ and a map $\bar{H}: X \times I \times T^{n-1} \rightarrow X$ by $\bar{H}\left(s, t_{1}, \ldots, t_{n-1}\right):=H\left(s, t_{1}, \ldots, t_{k-1}\right)$. It is clear that $[\bar{f} ; g] \in$ $G \sigma_{n}\left(X, x_{0}, G\right)$. Since $\left(X, x_{0}, G\right)$ is homotopically $n$-equivariant, it follows from Remark 3.6 that there exist an associated $G$-map $\bar{F}: X \times I \times T^{n-1} \rightarrow X$ to $\bar{f}$ such that

$$
\begin{aligned}
\bar{F}\left(x, 0, t_{1}, \ldots, t_{n-1}\right) & =x, \\
\bar{F}\left(x, 1, t_{1}, \ldots, t_{n-1}\right) & =g \cdot x \\
\bar{F}\left(x_{0}, s, t_{1}, \ldots, t_{n-1}\right) & =\bar{f}\left(s, t_{1}, \ldots, t_{n-1}\right)=f\left(s, t_{1}, \ldots, t_{k-1}\right) .
\end{aligned}
$$

Define a map $F: X \times I \times T^{k-1} \rightarrow X$ by

$$
F\left(x, s, t_{1}, \ldots, t_{k-1}\right):=\bar{F}\left(x, s, t_{1}, \ldots, t_{k-1}, 0, \ldots, 0\right) .
$$

Then it is clear that $F$ is an associated $G$-map to $f$. Hence $[f ; g]$ is homotopically $k$ equivariant. This completes the proof.

EXAMPLE 3.9. (a) Let $\mathbb{Z}_{m}=\langle\overline{1}\rangle$ act freely and properly discontinuously on $S^{1} \subset \mathbb{C}$ by $\overline{1} \cdot e^{i \theta}=e^{i\left(\theta+\frac{2 \pi}{m}\right)}$. By Lemma 3.2, it follows that $\left(\mathbb{Z}_{m}\right)_{0}=\mathbb{Z}_{m}$. Since $S^{1}$ is an $H$ space, it is Gottlieb-Fox and so $\tau_{n}\left(S^{1}\right)=G \tau_{n}\left(S^{1}\right) \cong \mathbb{Z}$ for any $n \geq 1$. Thus we may see that $\left(S^{1}, \mathbb{Z}_{m}\right)$ is Gottlieb-Rhodes. It can be easily seen that for any $n \geq 1, \sigma_{n}\left(S^{1}, 1, \mathbb{Z}_{m}\right)=$ $G \sigma_{n}\left(S^{1}, 1, \mathbb{Z}_{m}\right) \cong \mathbb{Z}$, which is generated by $[f ; g]$, where $f=\left\{e^{i \theta} \mid 0 \leq \theta \leq \frac{2 \pi}{m}\right\}$. Define a homotopy $H: S^{1} \times I \times T^{n-1} \rightarrow S^{1}$ by $H\left(e^{i \theta}, s, t_{1}, \ldots, t_{n-1}\right):=e^{i\left(\theta+\frac{2 \pi s}{m}\right)}$. It can be easily checked that $H$ is an associated $G$-map to $f$. Hence $\left(S^{1}, \mathbb{Z}_{m}\right)$ is homotopically equivariant.

(b) Let $n \geq 2$ and $m$ be positive integers. Consider $S^{2 n-1}$ as the unit sphere in $\mathbb{C}^{n}$, i.e., $S^{2 n-1}:=\left\{\left.\left(z_{1}, \ldots, z_{n}\right) \in \mathbb{C}^{n}|| z_{1}\right|^{2}+\cdots+\left|z_{n}\right|^{2}=1\right\}$. Then the map $\left(z_{1}, \ldots, z_{n}\right) \rightarrow$ $\left(e^{\frac{2 \pi i}{m}} z_{1}, \ldots, e^{\frac{2 \pi i}{m}} z_{n}\right)$ defines a free action of the finite cyclic group $\mathbb{Z}_{m}$ on $S^{2 n-1}$. By Lemma 3.2 , it follows that $\left(\mathbb{Z}_{m}\right)_{0}=\mathbb{Z}_{m}$. Since $\tau_{1}\left(S^{2 n-1}\right)=G \tau_{1}\left(S^{2 n-1}\right)=1$, we may see that $\sigma_{1}\left(S^{2 n-1}, \mathbb{Z}_{m}\right) \cong G \sigma_{1}\left(S^{2 n-1}, \mathbb{Z}_{m}\right) \cong \mathbb{Z}_{m}$. Moreover it can be seen that $G \sigma_{1}\left(S^{2 n-1}, \mathbb{Z}_{m}\right)$ is generated by $\left[f_{1} ; g\right]$, where $f_{1}=\left\{\left(e^{i \theta}, 0, \ldots, 0\right) \mid 0 \leq \theta \leq \frac{2 \pi}{m}\right\}$. Define a homotopy $H_{1}: S^{2 n-1} \times I \rightarrow S^{2 n-1}$ by

$$
H_{1}\left(\left(z_{1}, \ldots, z_{n}\right), s\right)=\left(e^{\frac{2 \pi s i}{m}} z_{1}, \ldots, e^{\frac{2 \pi s i}{m}} z_{n}\right) .
$$


It can be checked that $H_{1}$ is an associated $\mathbb{Z}_{m}$-map to $f_{1}$. Thus $\left(S^{2 n-1}, \mathbb{Z}_{m}\right)$ is homotopically 1 -equivariant. By the same argument, we may see that $G \sigma_{2 n-2}\left(S^{2 n-1}, \mathbb{Z}_{m}\right) \cong \mathbb{Z}_{m}$ which is generated by $\left[f_{2 n-2} ; g\right]$, where $f_{2 n-2}: I \times T^{2 n-3} \rightarrow S^{2 n-1}$ is defined by $f_{2 n-2}\left(s, t_{1}, \ldots\right.$, $\left.t_{2 n-3}\right):=f_{1}(s)$. Define a homotopy $H_{2 n-2}: S^{2 n-1} \times I \times T^{2 n-3} \rightarrow S^{2 n-1}$ by

$$
H_{2 n-2}\left(\left(z_{1}, \ldots, z_{n}\right), s, t_{1}, \ldots, t_{2 n-3}\right):=H_{1}\left(\left(z_{1}, \ldots, z_{n}\right), s\right) .
$$

It can be seen that $H_{2 n-2}$ is an associated $\mathbb{Z}_{m}$-map to $f_{2 n-2}$. Thus we can conclude that $\left(S^{2 n-1}, \mathbb{Z}_{m}\right)$ is also homotopically $(2 n-2)$-equivariant. Moreover it follows from Proposition 3.8 that $\left(S^{2 n-1}, \mathbb{Z}_{m}\right)$ is homotopically $k$-equivariant for any $1 \leq k \leq 2 n-2$.

It is of interest to find out which spaces with free and properly discontinuous $G$-action are homotopically $n$-equivariant owing to Theorem 3.11, which is one of the main results of this paper.

The following lemma is immediate and we state it without its proof.

LEMMA 3.10. For any $n \geq 1$, let $i_{n}: I \times T^{n-1} \hookrightarrow I \times T^{n}$ and $\pi_{n}: I \times T^{n} \rightarrow I \times T^{n-1}$ be the map defined by

$$
\begin{aligned}
& i_{n}:\left(s, t_{1}, \ldots, t_{n-1}\right) \rightarrow\left(s, t_{1}, \ldots, t_{n-1}, 0\right) \\
& \pi_{n}:\left(s, t_{1}, \ldots, t_{n-1}, t_{n}\right) \rightarrow\left(s, t_{1}, \ldots, t_{n-1}\right) .
\end{aligned}
$$

The maps $i_{n}$ and $\pi_{n}$ induces the following injective homomorphisms and surjective homomorphisms:
(a) $i_{n *}: G \sigma_{n+1}\left(X, x_{0}, G\right) \rightarrow G \sigma_{n}\left(X, x_{0}, G\right), \quad i_{n *}([f ; g])=\left[f \circ i_{n} ; g\right]$.
(b) $i_{n *}: G \tau_{n+1}\left(X, x_{0}\right) \rightarrow G \tau_{n}\left(X, x_{0}\right), \quad i_{n *}([f])=\left[f \circ i_{n}\right]$.
(c) $\pi_{n *}: G \sigma_{n}\left(X, x_{0}, G\right) \hookrightarrow G \sigma_{n+1}\left(X, x_{0}, G\right), \quad \pi_{n *}([f ; g])=\left[f \circ \pi_{n} ; g\right]$.
(d) $\pi_{n *}: G \tau_{n}\left(X, x_{0}\right) \hookrightarrow G \tau_{n+1}\left(X, x_{0}\right), \quad \pi_{n *}([f])=\left[f \circ \pi_{n}\right]$.

THEOREM 3.11. Let $G$ be a group which acts freely and properly discontinuously on a compactly generated Hausdorff space $X$ with a base point $x_{0}$. If $\left(X, x_{0}, G\right)$ is homotopically n-equivariant, then the map

$$
\Phi_{k}: G \sigma_{k}\left(X, x_{0}, G\right) \rightarrow G \tau_{k}\left(X / G, p\left(x_{0}\right)\right)
$$

defined by $\Phi_{k}([f ; g])=[p \circ f]$ is an isomorphism for all $1 \leq k \leq n$, where $p: X \rightarrow X / G$ is a regular covering projection.

Proof. We will show this by induction. Consider first the $n=1$ case. Take any $[f ; g] \in G \sigma_{1}\left(X, x_{0}, G\right)$. Since $\left(X, x_{0}, G\right)$ is homotopically $n$-equivariant, it follows from Proposition 3.8 and Remark 3.6 that there exists an affliated $G$-map $H: X \times I \rightarrow X$ to $f$ such that $H(x, 0)=x, H(x, 1)=g \cdot x$ and $H\left(x_{0}, t\right)=f(t)$. Define a map $K: X / G \times I \rightarrow$ $X / G$ by $K(p(x), t)=p \circ H(x, t)$. In order to see that $K$ is well-defined, suppose that $p\left(x_{1}\right)=p\left(x_{2}\right)$. Then $x_{1}=g \cdot x_{2}$ for some $g \in G$. Since $H$ is a $G$-map, it follows that

$$
\begin{aligned}
K\left(p\left(x_{1}\right), t\right) & =p \circ H\left(x_{1}, t\right)=p \circ H\left(g \cdot x_{2}, t\right) \\
& =p\left(g \cdot H\left(x_{2}, t\right)\right)=p\left(H\left(x_{2}, t\right)\right)=K\left(p\left(x_{2}\right), t\right) .
\end{aligned}
$$


Thus $K$ is well-defined continuous map. Since $K(p(x), 0)=p(x)=K(p(x), 1)$ and $K\left(p\left(x_{0}\right), t\right)=p \circ f(t)$, it follows that $[p \circ f] \in G \tau_{1}(X / G)$. Suppose now that $\left[f_{1} ; g\right]=$ $\left[f_{2} ; g\right]$. It is clear that $f_{1} \simeq f_{2}$, and so $\Phi\left(\left[f_{1} ; g\right]\right)=\left[p \circ f_{1}\right]=\left[p \circ f_{2}\right]=\Phi\left(\left[f_{2} ; g\right]\right)$. Hence we can define a well-defined homomorphism

$$
\Phi_{1}: G \sigma_{1}\left(X, x_{0}, G\right) \rightarrow G \tau_{1}\left(X / G, p\left(x_{0}\right)\right)
$$

by $\Phi_{1}([f ; g])=[p \circ f]$. Recall that $p_{*}: \sigma_{1}\left(X, x_{0}, G\right) \rightarrow \tau_{1}\left(X / G, p\left(x_{0}\right)\right)$ defined by $p_{*}([f ; g])=[p \circ f]$ is an isomorphism ([16], [8, Theorem 1.2]). Thus a restriction homomorphism $\Phi=\left.p_{*}\right|_{G \sigma_{1}\left(X, x_{0}, G\right)}$ is a monomorphism.

In order to show that $\Phi_{1}$ is surjective, take any $[f] \in G \tau_{1}\left(X / G, p\left(x_{0}\right)\right)$. Then there exists a cyclic homotopy $F: X / G \times I \rightarrow X / G$ such that $F\left(p\left(x_{0}\right), t\right)=f(t)$. For each $p(x) \in X / G$, define $f_{p(x)}: I \rightarrow X / G$ by $f_{p(x)}(t):=F(p(x), t)$. Since $f_{p(x)}(t)$ is a loop at $p\left(x_{0}\right)$ in $X / G$, it follows from the lifting theorem ([14, Lemma 54.1]) that for any $x \in p_{\tilde{f}^{-1}}(p(x))$, there exists a unique lifting path $\widetilde{f}_{p(x)}: I \rightarrow X$ such that $p \circ \widetilde{f}_{p(x)}=f_{p(x)}$ and $\widetilde{f}_{p(x)}(0)=x$. Now define $H: X \times I \rightarrow X$ by $H(x, t):=\widetilde{f}_{p(x)}(t)$. It is clear that $H$ is well-defined and continuous, since $I$ is locally compact Hausdorff (cf. [14]). Note that $H(x, 0)=x$ and $H(x, 1)=g \cdot x$ for some $g \in G$. Thus $H$ is a homotopy between $1_{X}$ and $g \cdot 1_{X}$. and thereby $\left[\widetilde{f}_{p\left(x_{0}\right)} ; g\right] \in G \sigma_{1}\left(X, x_{0}, G\right)$. Therefore

$$
\Phi_{1}\left(\left[\tilde{f}_{p\left(x_{0}\right)} ; g\right]\right)=\left[p \circ \tilde{f}_{p\left(x_{0}\right)}\right]=\left[f_{p\left(x_{0}\right)}\right]=[f] .
$$

Hence $\Phi_{1}$ is surjective and thereby $G \sigma_{1}\left(X, x_{0}, G\right) \cong G \tau_{1}\left(X / G, p\left(x_{0}\right)\right)$.

Now assume that $\Phi_{k}: G \sigma_{k}\left(X, x_{0}, G\right) \cong G \tau_{k}\left(X / G, p\left(x_{0}\right)\right)$ is true for $k<n$. From Lemma 3.10 we have the following commutative diagram:

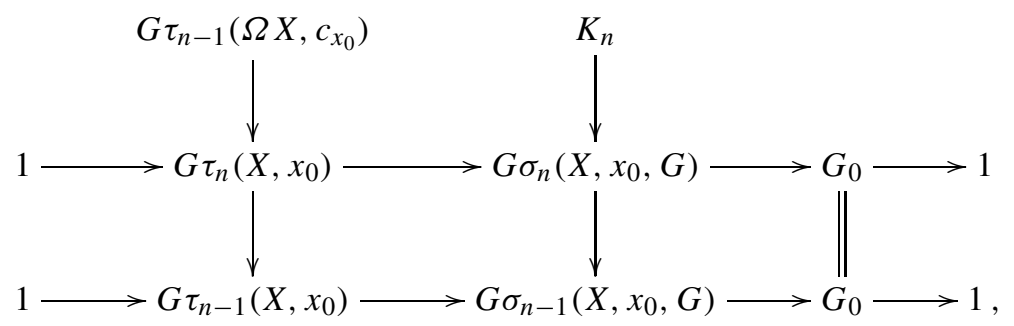

where $c_{x_{0}}$ is the constant loop at $x_{0}, G_{0}$ is the subgroup of $G$ consisting of elements $g$ considered as homeomorphisms of $X$ which are freely homotopic to $1_{X}$, and $K_{n}:=\operatorname{ker}\left(G \sigma_{n}\left(X, x_{0}\right.\right.$, $\left.G) \rightarrow G \sigma_{n-1}\left(X, x_{0}, G\right)\right)$. It can be easily seen that $G \tau_{n-1}\left(\Omega X, x_{0}\right) \cong K_{n}$. Note that $G \tau_{n}\left(X, x_{0}\right) / G \tau_{n-1}\left(\Omega X, c_{x_{0}}\right) \cong G \tau_{n-1}\left(X, x_{0}\right)$. Note also that

$$
K_{n} \cong G \tau_{n-1}\left(\Omega X, c_{x_{0}}\right)=\tau_{n-1}\left(\Omega X, c_{x_{0}}\right) \cong \prod_{i=2}^{n} \pi_{i}\left(X, x_{0}\right)^{\alpha_{i}} .
$$

Consider the following commutative diagram: 


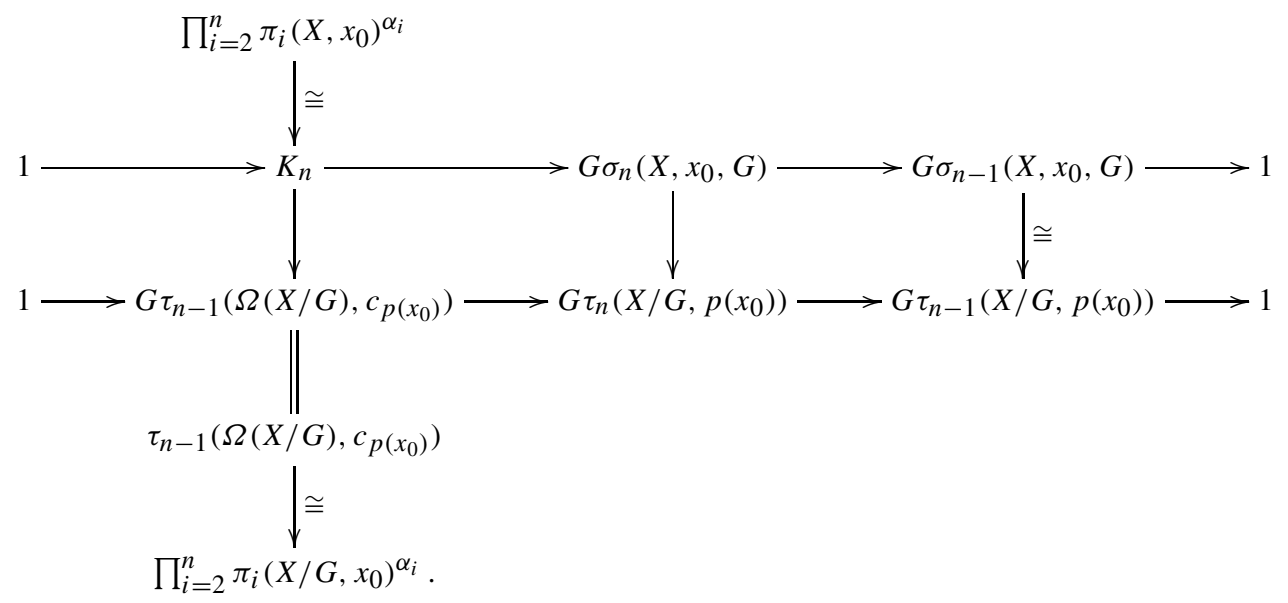

Since $\pi_{i}(X)=\pi_{i}(X / G)$ for $i \geq 2$, we have $G \tau_{n-1}\left(\Omega(X / G), c_{x_{0}}\right) \cong K_{n}$. Hence $\Phi_{n}$ : $G \sigma_{n}\left(X, x_{0}, G\right) \rightarrow G \tau_{n}\left(X / G, p\left(x_{0}\right)\right)$ is an isomorphism by the five lemma. This completes the proof.

COROLLARY 3.12. Let $G$ be a group which acts freely and properly discontinuously on a compactly generated Hausdorff space $X$ with a base point $x_{0}$. If $\left(X, x_{0}, G\right)$ is homotopically equivariant, then the map

$$
\Phi_{n}: G \sigma_{n}\left(X, x_{0}, G\right) \rightarrow G \tau_{n}\left(X / G, p\left(x_{0}\right)\right)
$$

defined by $\Phi_{n}([f ; g])=[p \circ f]$ is an isomorphism for all $n$, where $p: X \rightarrow X / G$ is a regular covering map.

PROOF. This follows immediately from Theorem 3.11.

As we mentioned in preliminaries, Gottlieb-Rhodes groups $G \sigma_{n}\left(X, x_{0}, G\right)$ are not abelian in general. But, it follows immediately from Theorem 3.11 that for a compactly generated Hausdorff based $G$-space $\left(X, x_{0}, G\right)$ with free and properly discontinuous $G$-action, if $\left(X, x_{0}, G\right)$ is homotopically $n$-equivariant, then $G \sigma_{n}\left(X, x_{0}, G\right)$ is abelian.

COROLLARY 3.13. Let $G$ be a group which acts freely and properly discontinuously on a compactly generated Hausdorff space $X$ with a base point $x_{0}$. If $\left(X, x_{0}, G\right)$ is homotopically n-equivariant, then $G \sigma_{n}\left(X, x_{0}, G\right)$ is abelian.

EXAMPLE 3.14. Let $n$ and $m$ be positive integers which are greater than or equal to 2. Consider $S^{2 n-1}$ with free and properly discontinuous $\mathbb{Z}_{m}$-action in Example 3.9. Then $\left(S^{2 n-1}, \mathbb{Z}_{m}\right)$ is homotopically $k$-equivariant for any $1 \leq k \leq 2 n-2$ by Example 3.9. Hence we see that $G \sigma_{k}\left(S^{2 n-1}, \mathbb{Z}_{m}\right) \cong G \tau_{k}\left(S^{2 n-1} / \mathbb{Z}_{m}\right)$ for all $1 \leq k \leq 2 n-2$ by Theorem 3.11 . Therefore we conclude that $G \sigma_{k}\left(S^{2 n-1}, \mathbb{Z}_{m}\right)$ is abelian for all $1 \leq k \leq 2 n-2$ by Corollary 3.13. Similarly we can show that $G \sigma_{k}\left(S^{1}, \mathbb{Z}_{m}\right) \cong G \tau_{k}\left(S^{1} / \mathbb{Z}_{m}\right)$ and $G \sigma_{k}\left(S^{1}, \mathbb{Z}_{m}\right)$ is abelian for all $k \geq 1$ by Example 3.9, Theorem 3.11, and Corollary 3.13. 
COROLLARY 3.15. Let $G$ be a group which acts freely and properly discontinuously on a compactly generated Hausdorff path-connected space $X$ with a base point $x_{0}$. Let $n$ be any positive integer. If $\left(X, x_{0}, G\right)$ is homotopically $n$-equivariant, then $X / G$ is $n$-Gottlieb if and only if $X$ is $n$-Gottlieb-Rhodes. Thus if $\left(X, x_{0}, G\right)$ is homotopically equivariant, then $X / G$ is Gottlieb if and only if $X$ is Gottlieb-Rhodes.

PROOF. Recall that a compactly generated Hausdorff path connected space $X$ is $n$ Gottlieb if and only if it is $n$-Gottlieb-Fox. Note that the isomorphisms $\sigma_{k}(X, G) \cong \tau_{k}(X / G)$ and $G \sigma_{k}(X, G) \cong G \tau_{k}(X / G)$ are given by the same map $\Phi_{k}$. Thus we may see from Theorems 3.1 and 3.11 that

$X / G$ is $n$-Gottlieb if and only if $X / G$ is $n$-Gottlieb-Fox

$$
\begin{aligned}
& \text { if and only if } \tau_{k}(X / G)=G \tau_{k}(X / G) \text { for all } 1 \leq k \leq n \\
& \text { if and only if } \sigma_{k}(X, G)=G \sigma_{k}(X, G) \text { for all } 1 \leq k \leq n \\
& \text { if and only if } X \text { is } n \text {-Gottlieb-Rhodes. }
\end{aligned}
$$

Hence we have the desired result.

4. Realization problems. By definition, an extension of a group $N$ by a group $G$ is a short exact sequence of groups $1 \rightarrow N \rightarrow \Gamma \rightarrow G \rightarrow 1$. Two extensions $1 \rightarrow N_{1} \rightarrow \Gamma_{1} \rightarrow$ $G_{1} \rightarrow 1$ and $1 \rightarrow N_{2} \rightarrow \Gamma_{2} \rightarrow G_{2} \rightarrow 1$ are said to be isomorphic if there are isomorphisms $N_{1} \rightarrow N_{2}, G_{1} \rightarrow G_{2}$ and $\Gamma_{1} \rightarrow \Gamma_{2}$ such that the following diagram commutes:

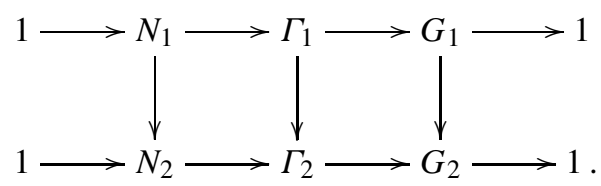

Definition 4.1 ([13, Definition 2.1]). Let $\theta_{1}$ and $\theta_{2}$ be actions of groups $G_{1}$ and $G_{2}$ on spaces $X_{1}$ and $X_{2}$, respectively. The action $\theta_{1}$ is said to be homotopy conjugate to $\theta_{2}$, denoted by $\theta_{1} \sim \theta_{2}$, if there exists a homotopy equivalence $h: X_{1} \rightarrow X_{2}$ and an isomorphism $\varphi: G_{1} \rightarrow G_{2}$ such that $h \circ \theta_{1}(G)=\theta_{2}(\varphi(g)) \circ h$ for all $g \in G_{1}$.

REMARK 4.2. If we denote the actions $\theta_{1}$ and $\theta_{2}$ by $\cdot$ and $*$, respectively, then the condition above in Definition 4.1 can be rephrased as follows: $h(g \cdot x)=\varphi(g) * h(x)$ for all $g \in G_{1}$ and all $x \in X_{1}$.

LEMMA 4.3. Let $\theta_{1}$ and $\theta_{2}$ be free and properly discontinuous actions of groups $G_{1}$ and $G_{2}$ on path-connected spaces $X_{1}$ and $X_{2}$, respectively. Assume that $\theta_{1}$ is homotopy conjugate to $\theta_{2}$, then the short exact sequences $1 \rightarrow \pi_{1}\left(X_{1}\right) \rightarrow \pi_{1}\left(X_{1} / G_{1}\right) \rightarrow G_{1} \rightarrow 1$ and $1 \rightarrow \pi_{1}\left(X_{2}\right) \rightarrow \pi_{1}\left(X_{2} / G_{2}\right) \rightarrow G_{2} \rightarrow 1$ are isomorphic so that the following diagram commutes: 


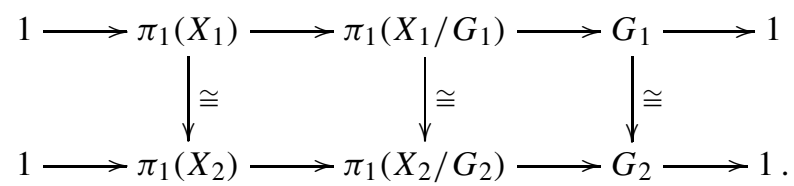

ProOF. It can be proved by the same argument as in [13, Lemma 2.1].

LEMMA 4.4. Let $\theta_{1}$ and $\theta_{2}$ be free actions of groups $G_{1}$ and $G_{2}$ on $C W$-complexes $X_{1}$ and $X_{2}$ of the type $K(N, 1)$, respectively. Assume that there exist isomorphisms $\varphi: G_{1} \rightarrow G_{2}$ and $\xi: \pi_{1}\left(X_{1} / G_{1}\right) \rightarrow \pi_{1}\left(X_{2} / G_{2}\right)$ such that the following diagram commutes:

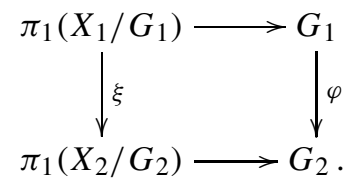

Then $\theta_{1}$ is homotopy conjugate to $\theta_{2}$ by the isomorphism $\varphi$ and a homotopy equivalence $h: X_{1} \rightarrow X_{2}$ such that $\xi \circ p r_{1 *}=p r_{2 *} \circ h_{*}$, where $\operatorname{pr}_{i *}: \pi_{1}\left(X_{i}\right) \rightarrow \pi_{1}\left(X_{i} / G_{i}\right)$.

PROOF. It can be proved by the same argument as in [13, Lemma 2.2].

LEMMA 4.5. Let $1 \rightarrow N \rightarrow \Gamma \rightarrow G \rightarrow 1$ be an exact sequence of groups. Then there exist a $K(N, 1)$-complex $X$ and a free action $\theta$ of $G$ on $X$ such that the following diagram commutes:

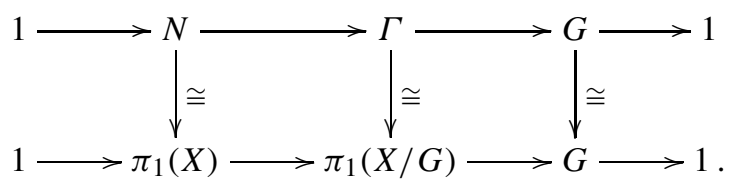

Here $X / G$ is a $K(\Gamma, 1)$-complex.

PROOF. It can be proved by the same argument as in [13, Lemma 2.3].

THEOREM 4.6. Let $1 \rightarrow N \rightarrow \Gamma \rightarrow G \rightarrow 1$ be an exact sequence of groups. Then there is a one-to-one correspondence between the following two sets:

(a) the set of all free actions up to homotopy conjugation of $G$ on $K(N, 1)$-complexes,

(b) the set of all classes of isomorphic extensions of $N$ by $G$.

PROOF. It follows from Lemmas 4.3, 4.4, and 4.5.

DEFINITION 4.7. Let $n$ be a positive integer.

(1) An exact sequence of groups $1 \rightarrow N \rightarrow \Gamma \rightarrow G \rightarrow 1$ is said to be $n$-Rhodes-Fox realizable if there exists a path-connected space $X$ with $G$-action such that $1 \rightarrow \tau_{n}(X) \rightarrow$ $\sigma_{n}(X, G) \rightarrow G \rightarrow 1$ is isomorphic to $1 \rightarrow N \rightarrow \Gamma \rightarrow G \rightarrow 1$, i.e., the following diagram commutes: 


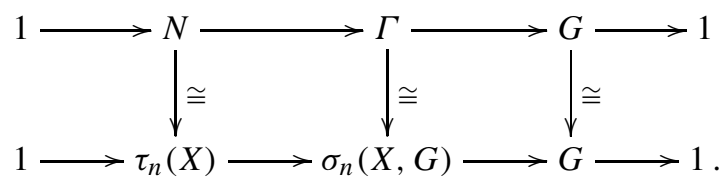

(2) An exact sequence of groups $1 \rightarrow N \rightarrow \Gamma \rightarrow Q \rightarrow 1$ with $N$ abelian is said to be $n$-(Gottlieb-Rhodes)-(Gottlieb-Fox) realizable if there exists a path-connected space $X$ with $G$-action such that $1 \rightarrow G \tau_{n}(X) \rightarrow G \sigma_{n}(X, G) \rightarrow G_{0} \rightarrow 1$ is isomorphic to $1 \rightarrow N \rightarrow$ $\Gamma \rightarrow Q \rightarrow$ 1, i.e., the following diagram commutes:

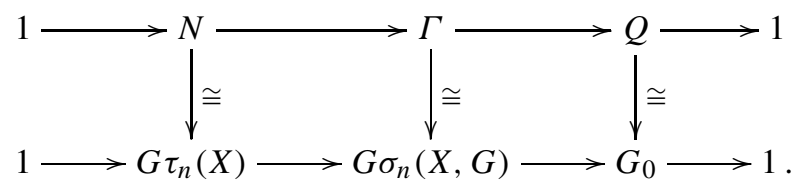

THEOREM 4.8. Every short exact sequence of groups $1 \rightarrow N \rightarrow \Gamma \rightarrow G \rightarrow 1$ is $n$-Rhodes-Fox realizable for any positive integer $n$. More precisely, there is a $K(N, 1)$ complex $X$ with free $G$-action such that for any positive integer $n$, the exact sequence of groups $1 \rightarrow \tau_{n}(X) \rightarrow \sigma_{n}(X, G) \rightarrow G \rightarrow 1$ is isomorphic to $1 \rightarrow N \rightarrow \Gamma \rightarrow G \rightarrow 1$.

Proof. From Lemma 4.5 it follows that there exists a free $G$-CW-complex $X$ of the type $K(N, 1)$ such that $1 \rightarrow \tau_{1}(X) \rightarrow \tau_{1}(X / G) \rightarrow G \rightarrow 1$ is isomorphic to $1 \rightarrow N \rightarrow$ $\Gamma \rightarrow G \rightarrow 1$. Note that $X / G$ is a $C W$-complex of the type $K(\Gamma, 1)$. From Theorem 3.1 and the definition of homomorphisms $\tau_{1}(X) \rightarrow \tau_{1}(X / G)$ and $\tau_{1}(X) \rightarrow \sigma_{1}(X, G)$ it follows that the following diagram commutes:

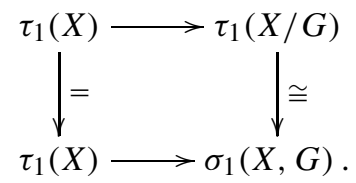

Thus we can show that $1 \rightarrow \tau_{1}(X) \rightarrow \sigma_{1}(X, G) \rightarrow G \rightarrow 1$ is isomorphic to $1 \rightarrow N \rightarrow$ $\Gamma \rightarrow G \rightarrow 1$. Since $X$ and $X / G$ are $C W$-complexex of the type $K(N, 1)$ and $K(\Gamma, 1)$, respectively, it follows from [9, Example 1.4] that $\tau_{n}(X) \cong N$ and $\tau_{n}(X / G) \cong \Gamma$ for all $n \geq 1$. By the definition of homomorphisms $\tau_{1}(X) \rightarrow \tau_{1}(X / G), \tau_{n}(X) \rightarrow \tau_{n}(X / G)$, and $\tau_{n}(X) \rightarrow \sigma_{n}(X / G)$ we may see that the following diagram commutes:

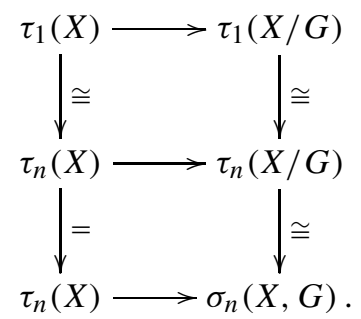


Hence we may conclude that $1 \rightarrow N \rightarrow \Gamma \rightarrow G \rightarrow 1$ is isomorphic to $1 \rightarrow \tau_{n}(X) \rightarrow$ $\sigma_{n}(X, G) \rightarrow G \rightarrow 1$.

COROLlary 4.9. Let $1 \rightarrow N \rightarrow \Gamma \rightarrow G \rightarrow 1$ be a short exact sequence of abelian groups. If the realizable free $G-C W$-complex $X$ in Theorem 4.8 admits a homotopically $n$ equivariant for a positive integer $n$, then the short exact sequence is $n$-(Gottlieb-Rhodes)(Gottlieb-Fox) realizable.

Proof. Note that $X$ and $X / G$ are $K(N, 1)$-complex and $K(\Gamma, 1)$-complex, respectively. Since any $H$-space is Gottlieb-Fox, it follows that $X$ and $X / G$ are Gottlieb-Fox. Thus $\tau_{n}(X)=G \tau_{n}(X)$ and $\tau_{n}(X / G)=G \tau_{n}(X / G)$. From Theorems 3.1 and 3.11 it follows that $\sigma_{n}(X, G) \cong \tau_{n}(X / G)=G \tau_{n}(X / G) \cong G \sigma_{n}(X, G)$. Hence we can conclude that the following diagram commutes so that $1 \rightarrow N \rightarrow \Gamma \rightarrow G \rightarrow 1$ is $n$-(Gottlieb-Rhodes)(Gottlieb-Fox) realizable:

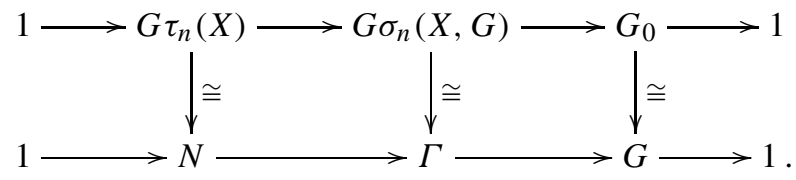

The following proposition implies that the $n$-th Rhodes groups and the $n$-th GottliebRhodes groups preserve the product. The versions of the first Rhodes groups and the first Gottlieb-Rhodes groups was proved in [16, Theorem 8] and [19, Theorem 1.9], respectively. Since their arguments can be applied to the higher dimensional case, we omit the proof of the following.

PROPOSITION 4.10. Let $G$ and $H$ be groups acting on spaces $X$ and $Y$, respectively. Then the following hold:

(1) $\sigma_{n}\left(X, x_{0}, G\right) \times \sigma_{n}\left(Y, y_{0}, H\right) \cong \sigma_{n}\left(X \times Y,\left(x_{0}, y_{0}\right), G \times H\right)$, where $G \times H$ acts componentwisely on the product space $X \times Y$.

(2) $G \sigma_{n}\left(X, x_{0}, G\right) \times G \sigma_{n}\left(Y, y_{0}, H\right) \cong G \sigma_{n}\left(X \times Y,\left(x_{0}, y_{0}\right), G \times H\right)$, where $G \times H$ acts componentwisely on the product space $X \times Y$.

EXAMPLE 4.11. It can be easily seen that there exist three non-isomorphic extensions of $\mathbb{Z}$ by $\mathbb{Z}_{2}$ as follows:

(a)

$$
1 \longrightarrow \mathbb{Z} \underset{1 \mapsto(1, \overline{0})}{\longrightarrow} \mathbb{Z} \times \mathbb{Z}_{2} \underset{(x, \overline{1}) \mapsto \overline{1}}{\longrightarrow} \mathbb{Z}_{2} \longrightarrow 1
$$

$$
1 \longrightarrow \mathbb{Z} \underset{1 \mapsto 2}{\longrightarrow} \mathbb{Z} \underset{1 \mapsto 1}{\longrightarrow} \mathbb{Z}_{2} \longrightarrow 1
$$

(c)

$$
1 \longrightarrow \mathbb{Z} \underset{1 \mapsto(1, \overline{0})}{\longrightarrow} \rtimes \mathbb{Z}_{2} \underset{(x, \overline{1}) \mapsto \overline{1}}{\longrightarrow} \mathbb{Z}_{2} \longrightarrow 1 .
$$


Let $X$ be the space $S^{1} \times S^{\infty}$, where $S^{\infty}=\widetilde{K\left(\mathbb{Z}_{2}, 1\right)}$ is an infinite dimensional sphere. Note that $X$ is a $C W$-complex of the type $K(\mathbb{Z}, 1)$. From Theorem 4.8 it follows that the exact sequences above are $n$-Rhodes-Fox realizable for any positive integer $n$. We give a realization of them as follows. Consider $S^{1}:=\left\{e^{i \theta} \mid 0 \leq \theta \leq 2 \pi\right\} \subset \mathbb{C}$. Let 1 be a base point of $S^{1}$ and $n$ a positive integer.

(a) Assume that $\mathbb{Z}_{2}$ acts on $S^{1}$ trivially and acts on $S^{\infty}$ by the reflection in the center. Then $\overline{1} \cdot(x, y)=(x,-y)$ where $(x, y) \in S^{1} \times S^{\infty}$. From Proposition 4.10 it follows that $\sigma_{n}\left(S^{1} \times S^{\infty}, \mathbb{Z}_{2}\right) \cong \tau_{n}\left(S^{1}\right) \times \sigma_{n}\left(S^{\infty}, \mathbb{Z}_{2}\right)$. By Theorem 3.1 we may see that $\sigma_{n}\left(S^{\infty}, \mathbb{Z}_{2}\right) \cong$ $\tau_{n}\left(K\left(\mathbb{Z}_{2}, 1\right)\right) \cong \mathbb{Z}_{2}$. Therefore $\sigma_{n}\left(S^{1} \times S^{\infty}, \mathbb{Z}_{2}\right) \cong \mathbb{Z} \times \mathbb{Z}_{2}$. Hence the first extension is $n$-Rhodes-Fox realizable by $X$ with the $\mathbb{Z}_{2}$-action above.

(b) Assume that $\mathbb{Z}_{2}$ acts on $S^{1}$ by $\overline{1} \cdot e^{i \theta}=e^{i(\theta+\pi)}$ and acts on $S^{\infty}$ trivially. By Proposition 4.10 , we have $\sigma_{n}\left(S^{1} \times S^{\infty}, \mathbb{Z}_{2}\right) \cong \sigma_{n}\left(S^{1}, \mathbb{Z}_{2}\right) \times \tau_{n}\left(S^{\infty}\right) \cong \sigma_{n}\left(S^{1}, \mathbb{Z}_{2}\right)$. Let $f$ be the upper half-circle in $S^{1}$ from 1 to -1 . Then $\overline{1} \cdot f$ is the lower half-circle from -1 to 1 . Thus we may see that if $\alpha$ is a loop based at 1 whose winding number is 1 , then $\alpha=f+\overline{1} \cdot f$. Hence every loop based at 1 is generated by $[f ; \overline{1}]$. It is clear that every element in $\sigma_{1}\left(S^{1}, 1, \mathbb{Z}_{2}\right)$ is generated by a linear combination of $f$ and $\alpha$. Thus every path in $\sigma_{1}\left(S^{1}, 1, \mathbb{Z}_{2}\right)$ is generated by $[f ; \overline{1}]$ and so $\sigma_{1}\left(S^{1}, 1, \mathbb{Z}_{2}\right) \cong \mathbb{Z}$. Hence $\sigma_{1}\left(S^{1} \times S^{\infty}, \mathbb{Z}_{2}\right) \cong \mathbb{Z}$. By the proof of Theorem 4.8, we may conclude that $\sigma_{n}\left(S^{1} \times S^{\infty}, \mathbb{Z}_{2}\right) \cong \mathbb{Z}$ and the second extension is $n$-Rhodes-Fox realizable by $X$ with the $\mathbb{Z}_{2}$-action above.

(c) Assume that $\mathbb{Z}_{2}$ acts diagonally on $S^{1}$ and $S^{\infty}$ by the reflection in the y-axis and by the reflection in the center, respectively. Let $s_{0}$ be a base point of $S^{\infty}$ and $h$ be an arbitrary path from $s_{0}$ to $\overline{1} \cdot s_{0}$. Note that $h+\overline{1} \cdot h$ is an inessential loop, since $S^{\infty}$ is contractible. It can be easily seen that $\left[\left(c_{1}, h\right), \overline{1}\right]$ is a torsion-element of order 2 , where $c_{1}$ is the constant loop in $S^{1}$ based at 1 . Thus we may see that $\sigma_{1}\left(S^{1} \times S^{\infty}, \mathbb{Z}_{2}\right)$ is isomorphic to $\mathbb{Z} \times \mathbb{Z}_{2}$ or $\mathbb{Z} \rtimes \mathbb{Z}_{2}$. Let $f_{u}$ be the upper half-circle and $f_{l}$ be the lower half-circle from 1 to -1 in $S^{1}$. It is clear that $f_{u}$ is not homotopic to $f_{l}$. Note that $f_{u}+\overline{1} \cdot f_{l}$ is a loop in $S^{1}$ based at 1 whose winding number is 1 , and $f_{l}+\overline{1} \cdot f_{u}$ is a loop based in $S^{1}$ at 1 whose winding number is -1 . It implies that $\sigma_{1}\left(S^{1} \times S^{\infty}, \mathbb{Z}_{2}\right)$ is not an abelian group and so $\sigma_{1}\left(S^{1} \times S^{\infty}, \mathbb{Z}_{2}\right) \cong \mathbb{Z} \rtimes \mathbb{Z}_{2}$. By the proof of Theorem 4.8, we may conclude that $\sigma_{n}\left(S^{1} \times S^{\infty}, \mathbb{Z}_{2}\right) \cong \mathbb{Z} \rtimes \mathbb{Z}_{2}$ and the third extension is $n$-Rhodes-Fox realizable by $X$ with the $\mathbb{Z}_{2}$-action above.

Recall that a transformation group $(X, G)$ admits a family of preferred traces at $x_{0}$ if and only if $G \sigma_{1}\left(X, x_{0}, G\right) \cong G \tau_{1}\left(X, x_{0}\right) \rtimes G_{0}$ if and only if $G \sigma_{1}\left(X, x_{0}, G\right) \cong G \tau_{1}\left(X, x_{0}\right) \times G_{0}$. By the same argument as in the proof of [20, Theorem 3, Theorem 4], we can show the following theorem without any difficulty.

THEOREM 4.12. For a transformation group $(X, G)$ and any $n \geq 1$, the following are equivalent:

(a) $(X, G)$ admits a family of preferred traces at $x_{0}$.

(b) $G \sigma_{n}\left(X, x_{0}, G\right) \cong G \tau_{n}\left(X, x_{0}\right) \rtimes G_{0}$.

(c) $G \sigma_{n}\left(X, x_{0}, G\right) \cong G \tau_{n}\left(X, x_{0}\right) \times G_{0}$. 
COROLlARY 4.13. Let $1 \rightarrow N \rightarrow \Gamma \rightarrow Q \rightarrow 1$ be a short exact sequence of groups with $N$ abelian. Suppose that $\Gamma$ is isomorphic to the semidirect product $N \rtimes Q$ but not the direct product $N \times Q$. Then for any positive integer $n$, there is no $K(N, 1)$-complex $X$ with free $G$-action such that $G_{0}=Q$ and $1 \rightarrow G \tau_{n}(X) \rightarrow G \sigma_{n}(X, G) \rightarrow G_{0} \rightarrow 1$ is isomorphic to $1 \rightarrow N \rightarrow \Gamma \rightarrow Q \rightarrow 1$.

Proof. By Theorem 4.8, there is a $K(N, 1)$-complex $X$ with free $Q$-action such that $\sigma_{n}(X, G) \cong \tau_{n}(X) \rtimes Q$. Since $N$ is abelian, it follows that $X$ is an $H$-space and so $\tau_{n}(X)=$ $G \tau_{n}(X)$. Suppose that there is a $K(N, 1)$-complex $X$ with free $G$-action such that $G_{0} \cong Q$ and $1 \rightarrow G \tau_{n}(X) \rightarrow G \sigma_{n}(X, G) \rightarrow G_{0} \rightarrow 1$ is isomorphic to $1 \rightarrow N \rightarrow \Gamma \rightarrow Q \rightarrow 1$. Then $G \sigma_{n}(X, G) \cong G \tau_{n}(X) \rtimes G_{0} \cong N \rtimes Q$. But by Theorem 4.12, $G \sigma_{n}(X, G) \cong G \tau_{n}(X) \times$ $G_{0} \cong N \times Q$, which makes a contradiction.

LEMMA 4.14. Let $\Gamma$ be an abelian group and $X$ a $K(\Gamma, 1)$-space. Then for any positive integer $n, \tau_{n}(X)=G \tau_{n}(X) \cong \Gamma$.

Proof. Since $\Gamma$ is abelian, any $K(\Gamma, 1)$-space $X$ is an $H$-space and so it is a GottliebFox space. Thus $\tau_{n}(X)=G \tau_{n}(X) \cong \prod_{i=1}^{n} G_{i}(X)^{\gamma_{i}}$, where $\gamma_{i}=\left(\begin{array}{c}n-1 \\ i-1\end{array}\right)$. Since $X$ is a $K(\Gamma, 1)$-space, the $n$-th homotopy group $\pi_{i}(X)$ is trivial for any $i \geq 2$. Thus $G_{i}(X)$ is also trivial for any $i \geq 2$. Therefore we may conclude that $\tau_{n}(X) \cong \prod_{i=1}^{n} G_{i}(X)^{\gamma_{i}} \cong G_{1}(X)=$ $\pi_{1}(X) \cong \Gamma$.

THEOREM 4.15. Let $\Gamma$ be a finitely generated abelian group and $n$ any positive integer. Then there exists a compact manifold $X$ such that $\tau_{n}(X) \cong \Gamma$.

Proof. Since $\Gamma$ is finitely generated abelian, it follows that there exist nonnegative integers $s$ and $t$ such that $\Gamma \cong\left(\bigoplus_{i=1}^{s} \mathbb{Z}\right) \oplus\left(\bigoplus_{j=1}^{t} \mathbb{Z}_{m_{j}}\right)$. Define $X$ as $\prod_{i=1}^{s} S_{i}^{n} \times \prod_{j=1}^{t} S_{j}^{2 n+1} /$ $\mathbb{Z}_{m_{j}}$, where $\mathbb{Z}_{m_{j}}$ acts freely on $S_{j}^{2 n+1}$ as in Example 3.9. Note that $\tau_{n}\left(S^{n}\right) \cong \mathbb{Z}$. Since $1 \rightarrow \tau_{n}\left(S^{2 n+1}\right) \rightarrow \sigma_{n}\left(S^{2 n+1}, \mathbb{Z}_{m_{j}}\right) \rightarrow \mathbb{Z}_{m_{j}} \rightarrow 1$ is exact and $\tau_{n}\left(S^{2 n+1}\right) \cong \pi_{n}\left(S^{2 n+1}\right)=1$, we may see that $\sigma_{n}\left(S^{2 n+1}, \mathbb{Z}_{m_{j}}\right) \cong \mathbb{Z}_{m_{j}}$. From Theorem 3.1 it follows that $\tau_{n}\left(S^{2 n+1} / \mathbb{Z}_{m_{j}}\right) \cong$ $\sigma_{n}\left(S^{2 n+1}, \mathbb{Z}_{m_{j}}\right) \cong \mathbb{Z}_{m_{j}}$. By Proposition 4.10, we can conclude that $\tau_{n}(X) \cong \Gamma$.

As we noted earlier, Oprea and Strom [15] proved the $n=1$ case of the following theorem. They showed that the higher dimensional version of the following theorem does not hold in ordinary Gottlieb groups. But for Gottlieb-Fox groups, we can show that for any positive integer $n$, every finitely generated abelian group is realized by the $n$-th Gottlieb-Fox group of a compact manifold,

THEOREM 4.16. Let $\Gamma$ be a finitely generated abelian group and $n$ any positive integer. Then there exists a compact manifold $X$ such that $G \tau_{n}(X) \cong \Gamma$.

Proof. The $n=1$ case was shown in [15, Theorem 3.1]. Let $n \geq 2$ be a positive integer and $\Gamma \cong\left(\bigoplus_{i=1}^{s} \mathbb{Z}\right) \oplus\left(\bigoplus_{j=1}^{t} \mathbb{Z}_{m_{j}}\right)$. Put $X:=\prod_{i=1}^{s} S_{i}^{1} \times \prod_{j=1}^{t} S_{j}^{2 n+1} / \mathbb{Z}_{m_{j}}$, where $\mathbb{Z}_{m_{j}}$ acts freely on $S_{j}^{2 n+1}$ as in Example 3.9. Note that $\tau_{n}\left(S^{1}\right)=G \tau_{n}\left(S^{1}\right) \cong \mathbb{Z}$ by Lemma 4.14, and $\sigma_{n}\left(S^{2 n+1}, \mathbb{Z}_{m_{j}}\right) \cong \mathbb{Z}_{m_{j}}$ as in the proof of Theorem 4.15. Since $G \tau_{n}\left(S^{2 n+1}\right) \leq \tau_{n}\left(S^{2 n+1}\right)=$ 
$\pi_{n}\left(S^{2 n+1}\right)=1$ and $1 \rightarrow G \tau_{n}\left(S^{2 n+1}\right) \rightarrow G \sigma_{n}\left(S^{2 n+1}, \mathbb{Z}_{m_{j}}\right) \rightarrow\left(\mathbb{Z}_{m_{j}}\right)_{0} \rightarrow 1$ is exact, it follows from Lemma 3.2 that $G \sigma_{2}\left(S^{3}, \mathbb{Z}_{m_{j}}\right) \cong \mathbb{Z}_{m_{j}}$. By Example 3.9 we may see that $\left(S^{2 n+1}, \mathbb{Z}_{m_{j}}\right)$ is homotopically $n$-equivariant. Thus it follows from Theorem 3.11 that

$$
G \tau_{n}\left(S^{2 n+1} / \mathbb{Z}_{m_{j}}\right) \cong G \sigma_{n}\left(S^{2 n+1}, \mathbb{Z}_{m_{j}}\right) \cong \mathbb{Z}_{m_{j}} .
$$

Hence we may see from Proposition 4.10 that

$$
\begin{aligned}
G \tau_{n}(X) & \cong G \tau_{n}\left(\prod_{i=1}^{s} S_{i}^{1}\right) \times G \tau_{n}\left(\prod_{j=1}^{t} S_{j}^{2 n+1} / \mathbb{Z}_{m_{j}}\right) \\
& \cong \bigoplus_{i=1}^{s} G \tau_{n}\left(S_{i}^{1}\right) \oplus \bigoplus_{j=1}^{t} G \tau_{n}\left(S_{j}^{2 n+1} / \mathbb{Z}_{m_{j}}\right) \\
& \cong \bigoplus_{i=1}^{s} \mathbb{Z} \oplus \bigoplus_{j=1}^{t} \mathbb{Z}_{m_{j}} \cong \Gamma .
\end{aligned}
$$

COROLlary 4.17. Let $\Gamma$ be a finitely generated abelian group and $n$ any positive integer. Then there exists a compact manifold $X$ which is a Gottlieb-Fox space such that $\tau_{n}(X)=G \tau_{n}(X) \cong \Gamma$.

PROOF. It follows immediately from Theorems 4.15 and 4.16 .

THEOREM 4.18. Let $\Gamma$ be a finitely generated abelian group. Then for any positive integer $n$, there exists a compact $G$-manifold $X$ such that the exact sequence of groups $1 \rightarrow$ $G \tau_{n}(X) \rightarrow G \sigma_{n}(X, G) \rightarrow G \rightarrow 1$ is isomorphic to $1 \rightarrow N \rightarrow \Gamma \rightarrow G \rightarrow 1$, where $N$ is a free abelian subgroup of $\Gamma$ and $G$ is a torsion subgroup of $\Gamma$.

PROOF. Let $\Gamma \cong\left(\bigoplus_{i=1}^{s} \mathbb{Z}\right) \oplus\left(\bigoplus_{j=1}^{t} \mathbb{Z}_{m_{j}}\right)$. Put $N:=\bigoplus_{i=1}^{s} \mathbb{Z}$ and $G:=\prod_{j=1}^{t} \mathbb{Z}_{m_{j}}$. Define $X$ as $\prod_{i=1}^{s} S_{i}^{1} \times \prod_{j=1}^{t} S_{j}^{2 n+1}$. Let $G$ act on each $S_{i}^{1}$ trivially and act freely on each $S_{j}^{2 n+1}$ as in Example 3.9. Note that $\tau_{n}\left(S^{1}\right)=G \tau_{n}\left(S^{1}\right) \cong \mathbb{Z}$. Since $G \tau_{n}\left(S^{2 n+1}\right) \leq \tau_{n}\left(S^{2 n+1}\right)$ $=\pi_{n}\left(S^{2 n+1}\right)=1$ and $1 \rightarrow G \tau_{n}\left(S^{2 n+1}\right) \rightarrow G \sigma_{n}\left(S^{2 n+1}, \mathbb{Z}_{m_{j}}\right) \rightarrow\left(\mathbb{Z}_{m_{j}}\right)_{0} \rightarrow 1$ is exact, it follows from Lemma 3.2 that $G \sigma_{n}\left(S^{2 n+1}, \mathbb{Z}_{m_{j}}\right) \cong \mathbb{Z}_{m_{j}}$. Thus we may conclude from Proposition 4.10 that

$$
\begin{aligned}
G \sigma_{n}(X, G) & \cong G \tau_{n}\left(\prod_{i=1}^{s} S_{i}^{1}\right) \times G \sigma_{n}\left(\prod_{j=1}^{t} S_{j}^{2 n+1}, G\right) \\
& \cong \bigoplus_{i=1}^{s} G \tau_{n}\left(S_{i}^{1}\right) \oplus \bigoplus_{j=1}^{t} G \sigma_{n}\left(S_{j}^{2 n+1}, \mathbb{Z}_{m_{j}}\right) \\
& \cong \bigoplus_{i=1}^{s} \mathbb{Z} \oplus \bigoplus_{j=1}^{t} \mathbb{Z}_{m_{j}} \cong \Gamma .
\end{aligned}
$$


Note that $G \tau_{n}(X) \cong \bigoplus_{i=1}^{s} G \tau_{n}\left(S_{i}^{1}\right) \oplus \bigoplus_{j=1}^{t} G \tau_{n}\left(S_{j}^{2 n+1}\right) \cong \bigoplus_{i=1}^{s} \mathbb{Z}$. Moreover it can be seen that $1 \rightarrow G \tau_{n}(X) \rightarrow G \sigma_{n}(X, G) \rightarrow G \rightarrow 1$ is isomorphic to $1 \rightarrow N \rightarrow \Gamma \rightarrow G \rightarrow 1$. This completes the proof.

Acknowledgment. The authors would like to thank the referee for useful comments and improvements of the earlier version of this paper. This research was supported by Basic Science Research Program through the National Research Foundation of Korea (NRF) funded by the Ministry of Education, Science and Technology (2012R1A1A2006395).

\section{REFERENCES}

[1] A. Adem And J. F. Davis, Topics in transformation groups, Handbook of geometric topology, NorthHolland, Amsterdam, 2002.

[2] S. Araki And M. Murayama, $G$-homotopy types of $G$ - $C W$-complexes and representation of $G$ cohomology theories, Publ. RIMS, Kyoto Univ. 14 (1978), 203-222.

[ 3 ] M. A. Armstrong, On the fundamental group of an orbit space, Proc. Cambridge Philos. Soc. 61 (1965), 639-646.

[ 4 ] K. S. Brown, Cohomology of groups, Graduate Texts in Mathematics, 87. Springer-Verlag, New York, 1994.

[ 5 ] R. H. Fox, Torus homotopy groups, Proc. Nat. Acad. Sci. U.S.A. 31 (1945), 71-74.

[ 6] R. H. Fox, Homotopy groups and torus homotopy groups, Ann. of Math. 49 (1948), 471-510.

[ 7 ] M. Golasiński, D. GonçCalves And P. Wong, Generalizations of Fox homotopy groups, Whitehead products and Gottlieb groups, Ukrainian Math. J. 57 (2005), 382-393.

[ 8 ] M. Golasiński, D. GonçAlves And P. Wong, Equivariant evaluation subgroups and Rhodes groups, Cah. Topol. Géom. Différ. Catég. 48 (2007), 55-69.

[9] M. Golasińs Ki, D. Gonçalves and P. Wong, On Fox spaces and Jacobi identities, Math. J. Okayama Univ. 50 (2008), 161-176.

[10] D. H. Gottlieb, A certain subgroup of the fundamental group, Amer. J. Math. 87 (1965), 840-856.

[11] D. H. GotTlieb, Evaluation subgroups of homotopy groups, Amer. J. Math. 91 (1969), 729-756.

[12] A. HATChER, Algebraic Topology, Cambridge University Press, Cambridge, 2002.

[13] L. V. LokUtSiEvSKII, Homotopy classification of actions of finite groups on aspherical spaces, J. Math. Sci. Soc. 177 (2011), 244-256.

[14] J. R. Munkres, Topology, 2nd ed., Prentice-Hall, Inc., Upper Saddle Riber, NJ, 2000.

[15] J. Oprea And J. Strom, On the realizability of Gottlieb groups, Contemp. Math. 519 (2010), 181-188.

[16] F. RHODES, On the fundamental group of a transformation group, Proc. London. Math. Soc. 16 (1966), 635650.

[17] F. RHodes, Homotopy groups of transformation groups, Canad. J. Math. 21 (1969), 1123-1136.

[18] T. том Dieck, Transformation groups, de Gruyter Studies in Mathematics, 8. Walter de Gruyter \& Co., Berlin, 1987.

[19] M. H. Woo AND Y. S. Yoon, Certain subgroups of homotopy groups of a transformation group, J. Korean Math. Soc. 20 (1983), 223-233.

[20] M. H. Woo, A representation of $E\left(X, x_{0}, G\right)$ in terms of $G\left(X, x_{0}\right)$, J. Korean Math. Soc. 23 (1986), 101-108. 
DEPARTMENT OF MATHEMATICS

SOGANG UNIVERSITY

SEOUL, 121-742

KOREA

E-mail address: crowd77@sogang.ac.kr jhjo@sogang.ac.kr

jmmoon87@sogang.ac.kr 